\title{
Improved routing strategies for Internet traffic delivery
}

\author{
Pablo Echenique, ${ }^{1,2}$ Jesús Gómez-Gardeñes, ${ }^{3,2}$ and Yamir Moreno ${ }^{2,1}$ \\ ${ }^{1}$ Departamento de Física Teórica, Universidad de Zaragoza, Zaragoza 50009, Spain \\ ${ }^{2}$ Instituto de Biocomputación y Física de Sistemas Complejos, Universidad de Zaragoza, Zaragoza 50009, Spain \\ ${ }^{3}$ Departamento de Física de la Materia Condensada, Universidad de Zaragoza, Zaragoza 50009, Spain
}

(Received 30 March 2004; published 15 November 2004)

\begin{abstract}
We analyze different strategies aimed at optimizing routing policies in the Internet. We first show that for a simple deterministic algorithm the local properties of the network deeply influence the time needed for packet delivery between two arbitrarily chosen nodes. We next rely on a real Internet map at the autonomous system level and introduce a score function that allows us to examine different routing protocols and their efficiency in traffic handling and packet delivery. Our results suggest that actual mechanisms are not the most efficient and that they can be integrated in a more general, though not too complex, scheme.
\end{abstract}

DOI: $10.1103 /$ PhysRevE.70.056105

PACS number(s): 89.75.Hc, 89.20.Hh, 89.75.Fb

Modern society increasingly depends on large communication networks such as the Internet. The need for information spreading pervades our lives and its efficient handling and delivery is becoming one of the most important practical problems. To this end, a suite of protocols for the dissemination of information from a given source to thousands of users has been developed in the last several years [1]. The Internet and other communication networks certainly work in a reliable way. However, both the physical network and the numbers of users are growing continuously. The scalability of current protocols and their performance for larger system sizes and heavier loads on the network are critical issues to be addressed in order to guarantee networks' functioning in the near future. From this perspective, one of the fundamental problems we face nowadays is to find optimal strategies for packet delivery between a given sending node and its destination host.

The above problem demands taking into account at least two factors. The first is related to the routers and servers capabilities-mainly, memory requirements and CPU processing time-needed for the different algorithms to operate efficiently. Second, it has been recently shown that the real architecture of communication networks determines many of their properties in front of dynamical processes such as the resilience to random failures and attacks and the spreading of virus and rumors [2-6]. The latter has been achieved in recent years by unraveling the complex patterns of interconnections that characterize seemingly diverse systems such as the Internet, the World Wide Web (WWW), and biological and social networks [7-9]. It turns out that most real networks can be described by growing models in which the number of nodes (or elements) forming the net increases with time and that the probability that a given node has $k$ connections to other nodes follows a power law $P_{k} \sim k^{-\gamma}$. This is the case of the Internet which shows a scale-free (SF) connectivity distribution with an exponent that has been estimated to be around $\gamma=2.2[1]$.

The aim of this paper is twofold. First, we show that the local properties of the networks on top of which a packet delivery process is taking place determine its efficiency. Then, we turn our attention to developing alternative strategies for Internet traffic routing by implementing a general stochastic protocol on top of a real Internet map at the autonomous system (AS) level. Through Monte Carlo simulations we show that the actual Internet's protocol is less efficient than one which combines knowledge of the topology and the traffic handled by the network at a local scale [10]. Our results might provide new hints for routing protocol designs as they are shown to need roughly the same capabilities of current routers.

In order to discuss how the local topological properties influence the efficiency of a given routing protocol, we use the network studied in Ref. [11]. In this model, the network is generated by considering the Barabási-Albert (BA) procedure [12] but introducing an affinity variable $f_{i}$ and a tolerance $\mu$, which determine the peers $j$ a new node can attach to. This is done by requiring that $f_{j} \in\left(f_{i} \pm \mu\right)$. This network shows the same global properties of the BA net regardless of the tolerance. However, depending on the value of $\mu$, other local properties, such as the clustering coefficient and correlations, differ from the original BA network.

Once we have a network whose local properties can be tuned, we proceed to define our basic routing protocol. In communication networks like the Internet, routers deliver data packets by ensuring that all routers converge to a best estimate of the path leading to each destination address. In other words, the routing process takes place following the criterion of the shortest available path length from a given source to its destination. Inspired by this protocol and with the aim of comparing its performance with other strategies, we define the following algorithm for packet delivery, which will be called the standard protocol. At the beginning, $p$ packets are created and both their destinations and the sources are chosen at random. In subsequent time steps, each node $i$ holding a packet sends it to its destination $j$ following the shortest path length between node $i$ and $j$ until all packets reach their destinations. That is, each packet is diverted in such a way that the distance $d_{i j}$, measured as the number of nodes one needs to pass by between $i$ and $j$, is minimized. In the case that there are more than one possible path, the choice is made at random. This recipe has been recently studied in generic network models $[13,14]$

The above procedure is repeated many times for a number 


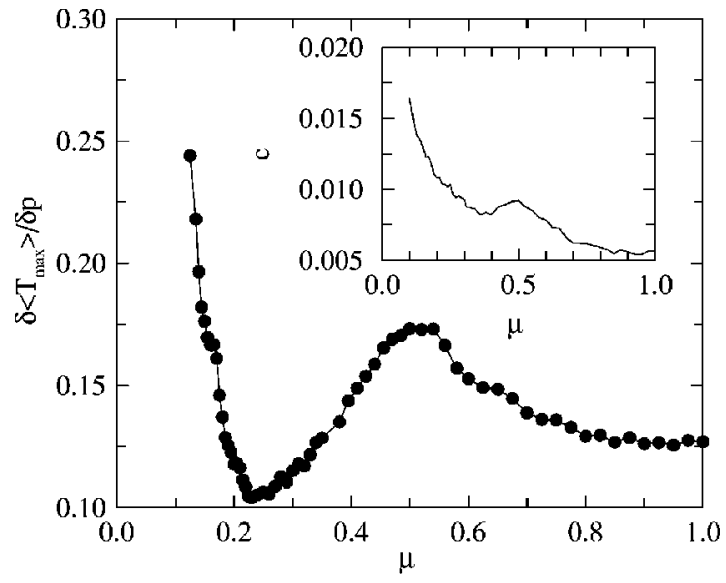

FIG. 1. $\partial\left\langle T_{\max }\right\rangle / \partial p$, as a function of the network parameter $\mu$. The figure illustrates the dependency of the standard routing protocol on the local properties of the network. The inset shows the variation of $c$ with $\mu$. The size of the network is $N=10^{4}$ nodes and $m_{0}=m=3$. The degree distribution is a power law with exponent equal to 3 . Note that the BA limit corresponds to $\mu=1$. See the text for further details.

of processes ranging from $p=1$ to at least $p=500$. Different realizations of the dynamics for the same $p$ are performed in order to average the relevant quantities. As a measure of the efficiency of the process, we have monitored $\left\langle T_{\max }\right\rangle$, the maximum time it takes for a packet to travel from its source to its destination, averaged over different realizations [15]. The numerical results show that this magnitude scales linearly with the number of processes, so that its derivative is a proper order parameter to characterize the routing performance. Figure 1 shows the slopes of the straight lines as a function of the control parameter $\mu$ which determines the local properties of the network. It is clear from the figure that the algorithm's outcome depends on the topological details of the network. For this network the average shortest path length $L$ is roughly the same as that of the BA network up to a value of $\mu$ around 0.2 . However, in this range of $\mu$ the efficiency has a maximum and a minimum. This implies that other properties are responsible for the behavior observednamely, the clustering coefficient $c$. For the network under analysis [11], it turns out that as $\mu$ is decreased from 1 (the BA limit) to smaller values, $c$ first slightly decreases below the BA value (accounting for the maximum) and then starts increasing up to values near the ones of real networks (see the inset in Fig. 1). From this perspective, it is then clear that the routing process is controlled by $c$ in the region where $L$ is constant. When $c$ increases, there appear new connections among the neighbors of a node and the number of shortest paths raises. Now, packets can circumvent more easily cogested nodes, thus making the standard protocol more efficient. When $c$ decreases, the opposite occurs. For very small $\mu, L$ diverges [11], leading to a bad performance of the protocol since the algorithm works on a shortest-pathdelivery basis. The crossover from the minimum to the divergence of $\partial\left\langle T_{\max }\right\rangle / \partial p$ is achieved in the parameter region where the interplay between $c$ and $L$ breaks down.

The preceding analysis shows that the routing protocol may be very sensitive to local details of the network on top of which the spreading process is taking place. It is then advisable to use real nets in order to obtain reliable results. To this end, we have used the Internet Autonomous System map at the Oregon route server dated 25 May 2001 [16]. It is worth stressing that each AS groups many routers together and the traffic carried by a node is the aggregation of the traffic generated at the internal routers and on individual endhost flows between the AS's.

The first modification of the routing mechanism is introduced by noting that the standard procedure does not take into account the traffic on the network. Specifically, a routing policy based on the shortest path between two given nodes neglects the queue in overloaded nodes which makes the process slower as the queue lengths become larger. That is, it may be more efficient to divert a packet through a larger but less congested path. Let us hence assume that a node $l$ is holding a packet that should be sent to a node $j$ and define an effective distance $d_{\text {eff }}^{i}$ from a neighboring node $i$ of $l$ to the destination $j$ as $d_{e f f}^{i}=d_{i}+c_{i}$, where $d_{i}$ is the shortest path between node $i$ and $j$ and $c_{i}$ is the number of processes (or packets) in the queue of $i$. The above definition, however, does not allow us a direct comparison with the standard procedure. It is convenient to redefine the effective distance as $d_{e f f}^{i}=h_{d} d_{i}+h_{c} c_{i}$ so that the limit $h_{c}=0$ contains the standard protocol. Furthermore, without loss of generality, we assume $h_{d}+h_{c}=1$. This algorithm will be called deterministic protocol henceforth. The rest of the algorithm remains the same as before; i.e., at each time step, the remaining packets are delivered in such a way that the path chosen is that which minimizes $d_{\text {eff }}^{i}$.

A first look at the dynamics shows that a protocol implemented in this way is more efficient than taking into account only the shortest-path criterion. In fact, $\left\langle T_{\max }\right\rangle$ departs from the linear behavior previously observed and is well below the straight line up to a high $p$. This behavior clearly depends on $h_{d}$, since it is straightforward to realize that if $h_{d}$ is zero, the packets are diverted following the less-loaded node regardless of the path length which results in an uncontrolled increase in the distance traveled by the packets from the sending nodes.

The above algorithm can be further generalized by including a probabilistic view. In other words, once we have determined the $d_{e f f}^{i}$ for all sending nodes $i$, we can allow for a stochastic choice of the paths. Hence, our third algorithm, referred to as stochastic protocol, considers a score function or "energy" $H_{i}=h_{d} d_{i}+\left(1-h_{d}\right) c_{i}$ and that the probability $\Pi_{i}$ that a packet is sent precisely through node $i$ is given by

$$
\Pi_{i}=\frac{e^{-\beta H_{i}}}{\sum e^{-\beta H_{j}}},
$$

where $\beta$ is the inverse of the temperature. In the limit $\beta$ $\rightarrow \infty$ (at zero temperature) we recover the deterministic protocol.

Figure 2 shows the dependence of $\left\langle T_{\max }\right\rangle$ on the number of packets $p$ for several values of $h_{d}$ in the deterministic limit of the model, which we found to be fulfilled for $\beta=20$. A dynamics which does not take into account the amount of 


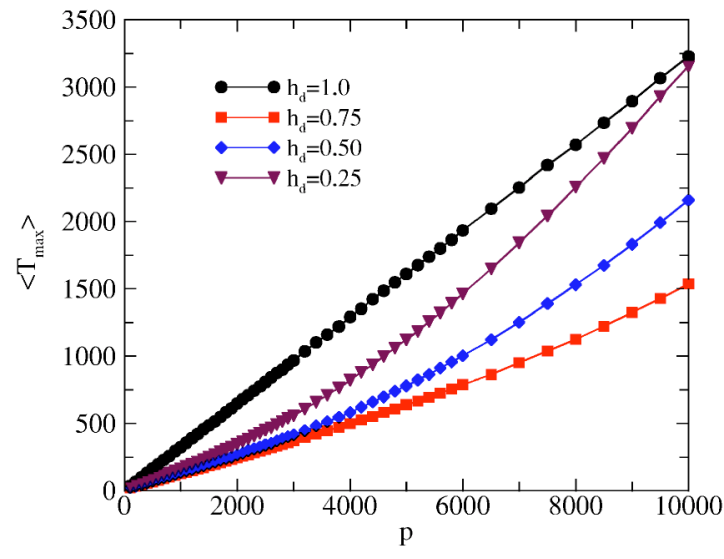

FIG. 2. (Color online) Dependence of $\left\langle T_{\max }\right\rangle$ on the number of initial packets $p$ in the deterministic limit of the model $(\beta=20)$ run on top of an AS Internet map made up of around 11000 nodes. Each point is an average over at least 200 realizations. The standard protocol corresponds to the limit $h_{d}=1$. Note that although the tendency of the curves is to cross the straight line as $p$ increases, there is an optimal value of $h_{d}$ such that interception would take place in the limit of very heavy traffic.

traffic handled by the neighbors of a sender node-straight line in Fig. 2-performs worse than the one which integrates both ingredients. However, this depends on the specific weight of each metric in $H_{i}$ and on $p$. In the regime where the traffic is not heavy (small $p$ values) all curves are below the standard protocol performance, but as the amount of traffic handled by the network increases, the deterministic protocol starts performing worse for a range of $h_{d}$ values. From the results, it seems that eventually, when the traffic increases too much, the curves cross the straight line indicating that at those limits the shortest path strategy is best suited. Note, however, that for $h_{d}=0.75$ the convergence of the two algorithms occurs for a very heavy load. Consequently, we can assert that there is an $h_{d}$ region where the combination of the two ingredients gives rise to the best performance. On the other hand, the existence of an optimal $h_{d}$ value distinct from zero can be understood by noting that a mechanism lacking some degree of path length information between the source and destination nodes of the packets performs badly because the packets travel along too large paths that do not compensate the time they would lose trapped in the queues of congested nodes.

The completely stochastic limit of the model corresponds to $\beta=0$. The performance of the protocol in this limit is, however, very bad. In fact, for an infinite temperature, all neighboring nodes of a given sender have the same probability to receive the message, and the dynamics becomes a random walk process. With no topological information about what are the destinations of the packets, they arrive to the receiver at longer times and the algorithm is the worst. For intermediate values of $\beta$, we have an stochastic dynamics in which topological and traffic information coexist. This is the case depicted in Fig. 3 for the same values of $h_{d}$ used in Fig. 2. As can be noted from the figure, the stochastic protocol increases $\left\langle T_{\max }\right\rangle$ by at least one order of magnitude as compared to the deterministic limit $(\beta=\infty)$. Moreover, the stan-

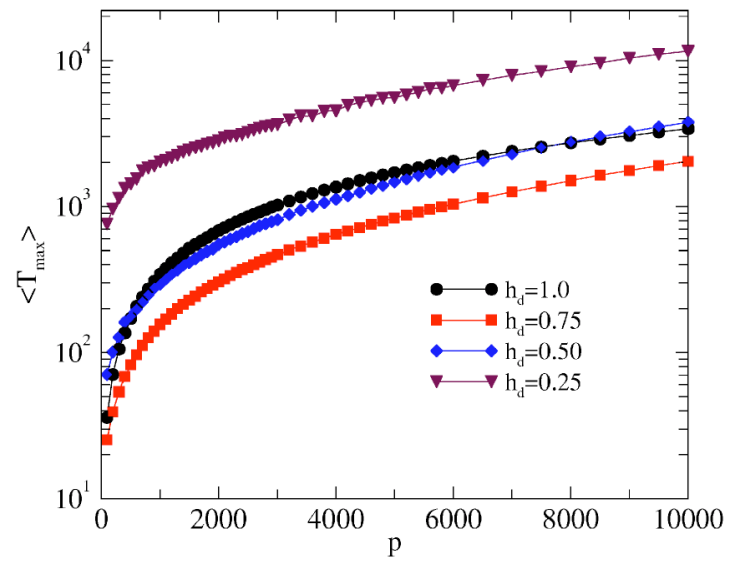

FIG. 3. (Color online) Dependence of $\left\langle T_{\max }\right\rangle$ on the number of packets, $p$, for a middle $\beta=5$ value. The network parameters are as in Fig. 2. In this case, the $h_{d}$ range in which the stochastic strategy performs better than the standard one is reduced.

dard approach seems to be the best choice for a wider range of $h_{d}$ values, although $h_{d}=0.75$ still performs better.

Figure 4 summarizes our results for different values of the control parameters $\beta$ and $h_{d}$. It turns out from the study of the whole phase diagram that the best algorithm is one which includes information about both path lengths and congestion at a local scale. Besides, the deterministic limit with $h_{d}$ $=0.75$ gives the best results for $\left\langle T_{\max }\right\rangle$. It would be worth noticing at this point that, although Fig. 4 was obtained in not too heavy traffic conditions, the results are consistent for larger values of $p$. Different tests allow us to conclude that the optimal value is $h_{d}=0.8 \pm 0.1$. In any case, this confirms that it would be possible to device more elaborated protocols with the aim of diminishing the time needed for a packet to spread through the network. In light of the present results, such a strategy may be implemented by also taking into account the amount of traffic handled by a local area of the network.

As suggested by Fig. 4, the best protocol is the deterministic one, which, on the other hand, should be more easy to implement in practice. The microscopic dynamics of the

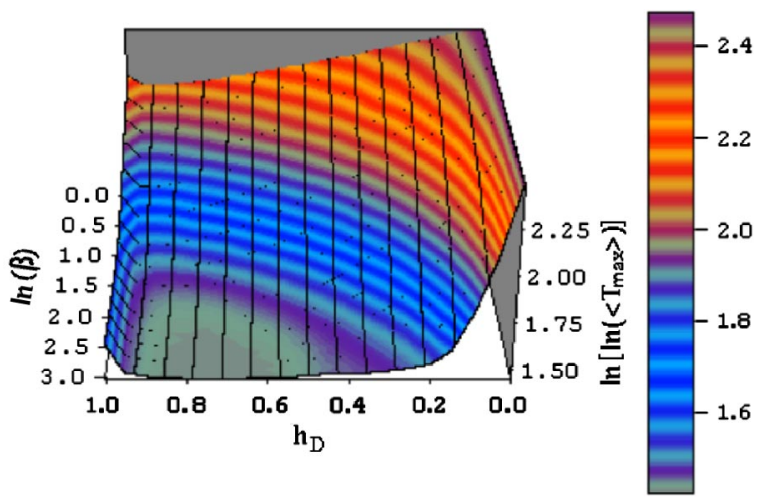

FIG. 4. (Color online) Phase diagram of the system's dynamics. The network parameters are as in Fig. 2. The number of processes is $p=500$. Calculations for higher $p$ show that the minimum of $\left\langle T_{\max }\right\rangle$ is also attained around $h_{d}=0.8 \pm 0.1$. 
routing process in this limit reveals that it is desirable that the routing process incorporate some knowledge of the node's queue lengths. However, the contribution in the score function of the second term should not weigh in excess. For small values of $h_{d}$-say, 0.25 - the algorithm performs better that the standard one because the packets do not pass by the hubs of the network, which are likely to be in the shortest path route to any node. Instead, they go around the hubs and $\left\langle T_{\max }\right\rangle$ is smaller. If $p$ is increased, the neighbors of the hubs also get congested. This leads to a situation in which the packets around a hub get trapped in its neighborhood, getting in and out from it, but without finding their routes to their destinations.

We finally point out that the improved strategies studied in this paper should not be hard to implement in practice. Actual protocols use the topological information used by the different variants explored. One would only need to provide further information on the traffic status in a local area. This could be done, for instance, by using the keep-alive messages that routers continuously exchange with their peers, though in this case there is some time delay due to the time scales of the routing process and the database or message exchanges.

In summary, we have studied alternative strategies for traffic delivery in complex heterogeneous networks. The results showed that the performance of the standard approach is sensitive to local topological changes. Specifically, the clustering properties may play a key role in message delivery. On the other hand, algorithms which integrate topological and traffic information have been shown to perform better that the standard protocol. Finally, we note that the present results could be extended to the Internet map at the router level which is statistically equivalent [1] to the AS map used here.

Y.M. thanks A. Vázquez for sharing his Internet database with us. P.E. and J.G-G. acknowledge financial support of the MECyD through FPU grants. Y.M. is supported by a BIFI research grant. This work has been partially supported by Spanish DGICYT Projects No. BFM2002-01798 and BFM2002-00113.
[1] R. Pastor-Satorras and A. Vespignani, Evolution and Structure of the Internet (Cambridge University Press, Cambridge, UK, 2004).

[2] R. Cohen, K. Erez, D. ben-Avraham, and S. Havlin, Phys. Rev. Lett. 85, 4626 (2000).

[3] D. S. Callaway, M. E. J. Newman, S. H. Strogatz, and D. J. Watts, Phys. Rev. Lett. 85, 5468 (2000).

[4] Y. Moreno, R. Pastor-Satorras, and A. Vespignani, Eur. Phys. J. B 26, 521 (2002).

[5] A. Vázquez and Y. Moreno, Phys. Rev. E 67, 015101(R) (2003).

[6] M. E. J. Newman, Phys. Rev. E 66, 016128 (2002).

[7] S. H. Strogatz, Nature (London) 410, 268 (2001).

[8] S. N. Dorogovtsev and J. F. F. Mendes, Evolution of Networks: From Biological Nets to the Internet and the WWW (Oxford University Press, Oxford, UK, 2003).

[9] Handbook of Graphs and Networks, edited by S. Bornholdt and H. G. Schuster (Wiley-VCH, Berlin, 2003).
[10] Several traffic-dependent routing protocols with rules different to those employed here have been studied by computer scientists without taking into account the actual topology of realworld nets.

[11] J. Gómez-Gardeñes and Y. Moreno, Phys. Rev. E 69, 037103 (2004).

[12] A.-L. Barabási and R. Albert, Science 286, 509 (1999).

[13] S. Valverde and R. V. Sóle, Physica A 312, 636 (2002).

[14] R. Guimera, A. Arenas, A. Díaz-Guilera, and F. Giralt, Phys. Rev. E 66, 026704 (2002).

[15] The choice of $\left\langle T_{\max }\right\rangle$ is arbitrary. One can also use $\left\langle T_{a v g}\right\rangle$ or $\left\langle T_{r m s}\right\rangle$, which leads to the same behaviors. We have chosen $\left\langle T_{\max }\right\rangle$ because the errors involved in its computation are the smallest of the three regardless of the algorithm.

[16] Available at http://topology.eecs.umich.edu/. Electric Engineering and Computer Science Department, University of Michigan, Topology Project. 